\title{
Extension Tip of Lift-Out Probe in FIB Systems
}

S. X. Wang

Micron Technology Inc., Boise, ID 83707, USA

The focused ion beam (FIB) lift-out procedure is commonly used to cut a small piece of sample from bulk material and lift it out within the scanning electron microscope $(\mathrm{SEM}) /$ FIB system $[1,2]$. In general, a metal tip is used for the in situ lift-out. Maintaining a sharp tip in the FIB is difficult because a portion of the tip will be consumed by every lift-out. Adding an extension tip to the existing lift-out probe overcomes this difficulty [3].

Figure 1 shows the schematic design of the extension tip as attached onto the original liftout probe. The commonly available Pt or W deposition in FIB systems can be used to "glue" the extension onto the probe. In practical use, the diameter of the extension tip is about $0.1-2 \mu \mathrm{m}$. The length of the extension tip varies based on the diameter of the tip, the required strength, and the convenience of the lift-out operation. It is normally made of electrically conductive material to avoid charging.

Any methods capable of producing micron-thin wires can be used to make the extension tip. For example, the FIB can be used to cut a small piece from a silicon wafer (as shown in Fig. 2), which can then be used as the extension tip. The metal lines in semiconductor devices may also be used as extension tips. Making the extension tip via FIB is preferred, however, because it offers more control over the shape, size, and orientation of the extension tip.

Using the silicon extension tip in a lift-out procedure demonstrates its strength and maneuverability. In Figure 3, the tip was used to lift out a sample with a width of a few microns.

The primary advantage of the extension tip is its easy replacement with a new, sharp tip. The extension tip makes lifting out a small sample_-including transmission electron microscopy-ready membrane - quite easy. The small diameter also makes the tip less rigid, thus reducing the chance of damaging the sample.

\section{References}

[1] L. A. Giannuzzi et. al., Mat. Res. Soc. Symp. Proc. 480 (1997) 19.

[2] L. R. Herlinger, S. Chevacharoenkul, D. C. Erwin, Proc. $22^{\text {nd }}$ Int. Symp. Test. Fail. Analy. (1996) 199.

[3] S. X. Wang, U.S. Patent Publication Serial Number 2005/0247886. 


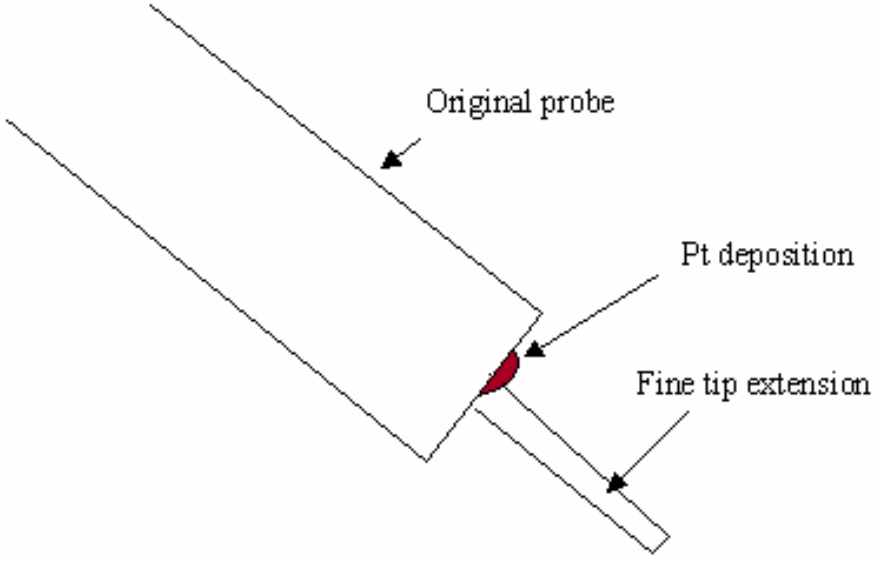

Fig. 1. Schematic diagram of the extension tip attached to the original probe.
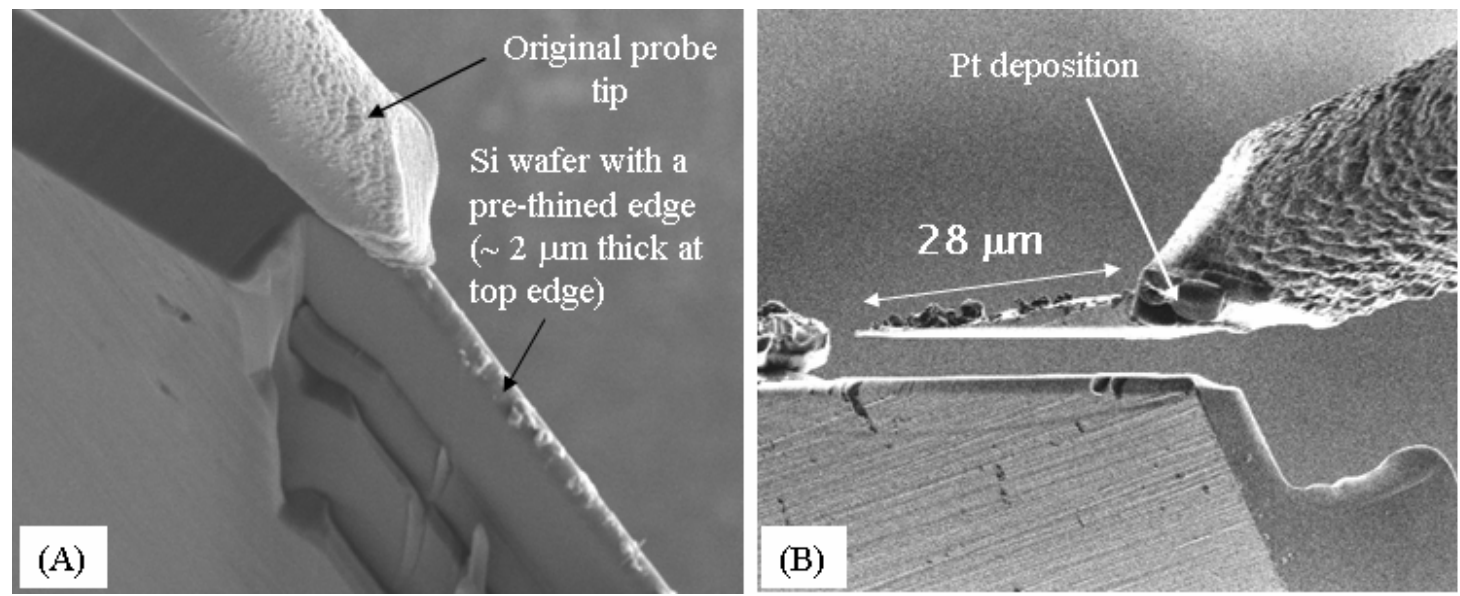

Fig. 2. (A) Insert the probe onto the thin edge of Si. The tip is glued onto Si by $\mathrm{Pt}$ deposition. (B) A fine Si-tip is cut free by ion beam can be retracted wth the original probe.
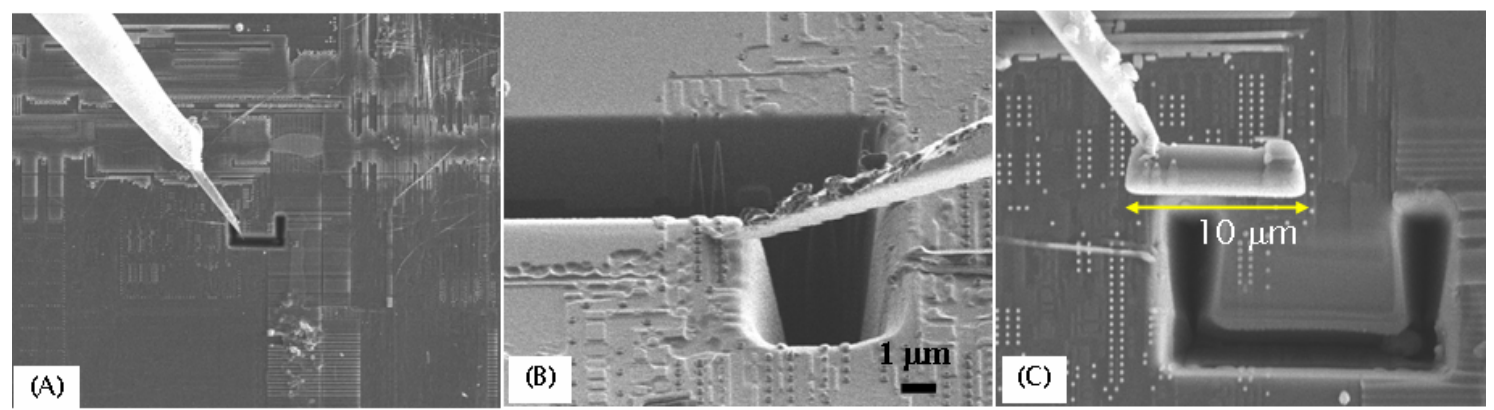

Fig. 3. (A) The extended tip is inserted into the sample surface in a FIB/SEM system. (B) A close-up view of the tip touching sample surface, showing the ease of maneuverability of the tip in a tight area. (3) A small piece of sample is glued onto the tip by Pt deposition, cut off by FIB, and lifted out by the extended tip. 